\title{
Untersuchungen zum Entstehungs- und Funktionszusammenhang von Dr. Heinrich Hoffmanns »Struwwelpeter»
}

\author{
Vorgelegt dem \\ Fachbereich I \\ der \\ Technischen Universität Berlin \\ zur Erlangung des Doktorgrades \\ von \\ MARIE-LUISE KÖNNEKER \\ aus \\ Remlingen
}


ISBN 978-3-476-99604-6

ISBN 978-3-476-99603-9 (eBook)

DOI 10.1007/978-3-476-99603-9

1. Berichter Prof. Dr. Volker Klotz

2. Berichter Prof. Dr. Norbert Miller

Tag der mündlichen Prüfung 31. 10. 1975 


\section{INHALTSVERZEICHNIS}

I. Der »Struwwelpeter« als Modell bürgerlicher Sozialisation und

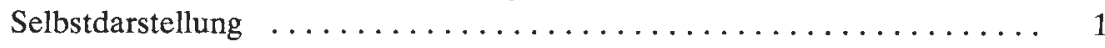

II. Zur Biographie Heinrich Hoffmanns als persönlicher Entstehungs-

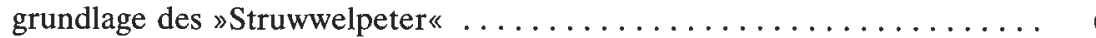

III. Der sozialgeschichtliche Kontext:

Zum Prozeß der Entfaltung einer spezifisch bürgerlichen Sozialisation und seiner Darstellung in der Erziehungs- und Kinderliteratur . . . . . . . . 16

1. Die sozioökonomische Struktur der bürgerlichen Kleinfamilie . . . . . . 16

2. Gegenmodell einer traditionsgelenkten Erziehung $\ldots \ldots \ldots \ldots \ldots \ldots \ldots 20$

3. Die erste Phase der bürgerlichen Erziehungsbewegung und ihre Spiegelung

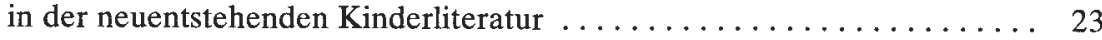

4. Differenzierung und Distanzierung von Erwachsenen und Kindern in ihrer Auswirkung auf die Sozialisation und die Emanzipation der bürgerlichen

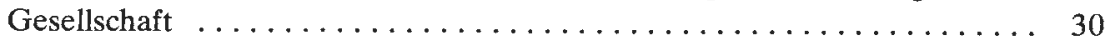

IV. Der »Struwwelpeter « als literarisch-ästhetischer Gegenstand der

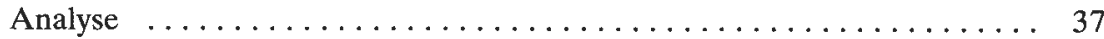

1. Zum Medium Bildergeschichte $\ldots \ldots \ldots \ldots \ldots \ldots \ldots \ldots \ldots \ldots \ldots \ldots$

2. Charakteristik der visuellen Darstellungsstruktur des $»$ Struwwelpeter $« \ldots .61$

2.1 Verschiedene Typen von Bildern und Bildfolgen . . . . . . . . . 61

2.2 Darstellung von Raum und Interieur $\ldots \ldots \ldots \ldots \ldots \ldots \ldots \ldots \ldots$

2.3 Formate und Größenverhältnisse $\ldots \ldots \ldots \ldots \ldots \ldots \ldots \ldots$

2.4 Mittel der Charakterisierung und Typisierung: Kleidung, Mimik, Gestik . . . . . . . . . . . . . . . . . . . 77

2.5. Zum Verhältnis von Bild und Text $\ldots \ldots \ldots \ldots \ldots \ldots \ldots \ldots \ldots$

3. Handlungsschemata und Mittel der Textgestaltung . . . . . . . . 86

3.1. Das dynamisch-emotionale Aufbauprinzip der Geschichten . . . . . . 86

3.2. Konfiguration der Personen und Kommunikationsstruktur . . . . . . 88

3.3. Rolle der Tiere . . . . . . . . . . . . . . . . . . . . . 89

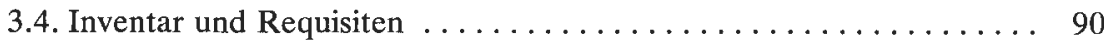

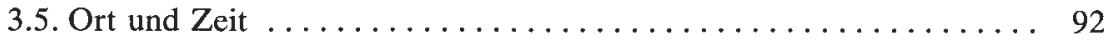

3.6. Zur sprachlichen Form der gereimten Geschichte für Kinder und ihrer Beziehung zur populären Literatur . . . . . . . . . . 93 
4. Bild-Text-Interpretation der einzelnen Geschichten . . . . . . . . 97

4.1. Die Geschichte vom bösen Friederich . . . . . . . . . . . . . . 97

4.2. Die gar traurige Geschichte mit dem Feuerzeug . . . . . . . . . . . . 104

4.3. Die Geschichte von den schwarzen Buben . . . . . . . . . . . . 112

4.4. Die Geschichte vom wilden Jäger . . . . . . . . . . . . . 115

4.5. Die Geschichte vom Daumen-Lutscher . . . . . . . . . . . . . . 118

4.6. Die Geschichte vom Suppen-Kaspar . . . . . . . . . . . . . . . . 122

4.7. Die Geschichte von dem Zappel-Philipp . . . . . . . . . . . . . . . 125

4.8. Die Geschichte vom Hanns Guck-in-die-Luft . . . . . . . . . . . . . 130

4.9. Die Geschichte vom fliegenden Robert . . . . . . . . . . . . . 135

V. Zur Genealogie der einzelnen im »Struwwelpeter» vermittelten Erziehungs-

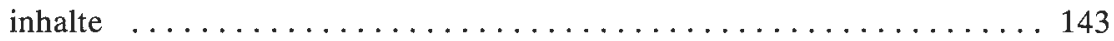

1. Hemmung der Gesamtmotorik als vordringliches Erziehungsziel . . . . . . 143

2. Strategien zur Unterdrückung der kindlichen Sexualität und die Bedeutung der sexuellen Symbolik im $»$ Struwwelpeter" . . . . . . . . . . . . . 146

3. Einschränkung oraler Bedürfnisbefriedigung $\ldots \ldots \ldots \ldots \ldots \ldots \ldots \ldots$

4. Kampf gegen Tierquälerei ....................... 160

5. Darstellung und Methode der Bestrafung: internalisierte und externalisierte

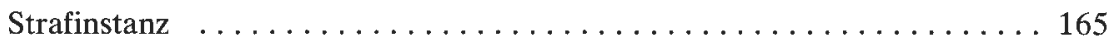

VI. Der politische Gehalt des »Struwwelpeter" und die Beziehung zur populären Bild- und Erzähltradition . . . . . . . . . . . . . . . . . . . 173

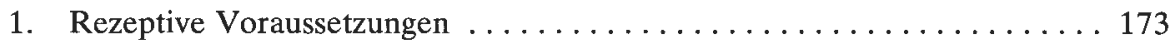

2. Das Motiv der >Verkehrten Welts als Abbild gesellschaftlicher Wider-

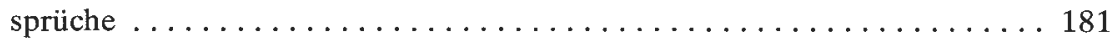

3. Traditionale und historisch-aktuelle Aspekte in der »Niklas«-Darstellung . 194

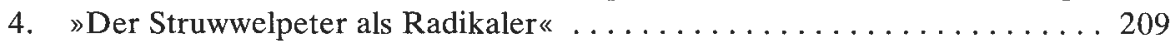

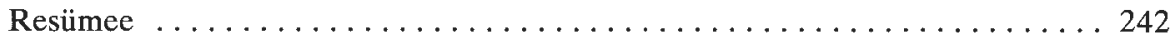

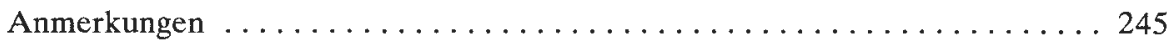

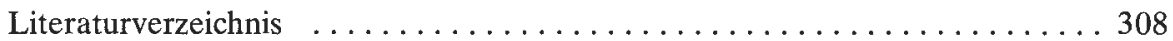

Hinweise zu Abbildungen und Bildquellen . . . . . . . . . . . 327

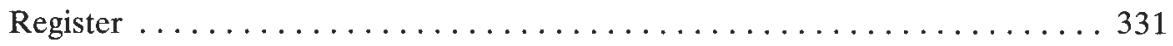

»Der Struwwelpeter « nach den ersten Auflagen (Leipzig 1957) . . . . . . . . 333 